\title{
Closed integral-differential equations of incompressible Navier-Stokes turbulent flow
}

\author{
Bohua Sun ${ }^{1}$ \\ ${ }^{1}$ Cape Peninsula University of Technology, Cape Town, South Africa*
}

(Dated: August 14, 2018)

\begin{abstract}
This paper showed that turbulence closure problem is not an issue at all. All mistakes in the literature regarding the numbers of unknown quantities in the Reynolds turbulence equations stem from the misunderstandings of physics of the Reynolds stress tensor, i.e., all literature has stated that the symmetric Reynolds stress tensor has six unknowns; however, it actually has only three unknowns, i.e., the three components of fluctuation velocity. We showed the integral-differential equations of the Reynolds mean and fluctuation equations have exactly eight equations, which equal to the numbers of quantities in total, namely, three components of mean velocity, three components of fluctuation velocity, one mean pressure and one fluctuation pressure. With this understanding, the closed Reynolds Navier-Stokes turbulence equations of incompressible flows were formulated. This study may help to solve the puzzle that has eluded scientists and mathematicians for centuries.
\end{abstract}

PACS numbers: 47.27.-i,47.27.Ak,47.27.E-

Keywords: Turbulence, the Reynolds stress tensor, turbulence closure problem

Turbulence is everywhere, controlling the drag on cars, airplanes, and trains, whilst dictating the weather through its influence on large-scale atmospheric and oceanic flows. Even solar flares are a manifestation of turbulence since they are triggered by vigorous motions on the surface of the sun. It is easy to be intrigued by a subject that pervades so many aspects of peoples' daily lives $[1-16]$.

The study of turbulence is not simple owing to its complex and forbidding mathematical descriptions, as well as the profound difficulties of inherent instabilities and even chaotic processes. People believe that turbulence prediction can be attained by understanding solutions to Navier-Stokes equations. However, understanding of the Navier-Stokes equations remains minimal, while there is still surprisingly little that can be predicted with relative certainty $[15,17,18]$. In respect of the turbulence problem, a myriad of tentative theories have been proposed, each with its own doctrines and beliefs, whilst often focused on particular experiments; however, there is not much in the way of a coherent theoretical framework $[1-8,10-16]$. Turbulence is a unique subject that engineers, mathematicians, and physicists tend to view in rather different ways. Many engineers promote the use of semi-empirical models of turbulence, while mathematicians advocate the use of purely statistical models [19-24], and the formalism of chaos theory and fractals [25-27].

*Electronic address: sunb@cput.ac.za
In 1972 a new chapter was launched in turbulence theory: Orszag and Patterson demonstrated that it was possible to perform direct numerical simulation (DNS) of a fully turbulent flow [28]. It is important to understand that DNS does not require any turbulence model to parameterize influence of the turbulent eddies. Rather, every eddy, from the largest to the smallest, is computed. Technically speaking, the turbulence can be solved by DNS if computers have infinite speed. However, a huge chasm remains between what the engineer needs to know, and what can be realized by DNS, using current computers. Even if DNS can assist to solve turbulence issues and problems, one still requires turbulence modelling to acquire a physical understanding of it.

Although there are different views about turbulence, there is a consensus that the deterministic Navier-Stokes equation probably contains all information relevant to turbulence [11]. It is believed that turbulence can be figured out once the Navier-Stokes equation is solved. Hence, scholars have been critical of the Navier-Stokes equation, and numerous works have been published as a result $[1-8,10-16]$.

In 1895 Reynolds published a seminal work on turbulence [29], in which he proposed that flow velocity $\boldsymbol{u}$ and pressure $p$ are decomposed into its time-averaged quantities, $\overline{\boldsymbol{u}}, t, \bar{p}$, and fluctuating quantities, $\boldsymbol{u}^{\prime}, p^{\prime}$; thus, the Reynolds decompositions are: $\boldsymbol{u}=\overline{\boldsymbol{u}}(\boldsymbol{x}, t)+\boldsymbol{u}^{\prime}(\boldsymbol{x}, t)$ and $p(\boldsymbol{x}, t)=\bar{p}(\boldsymbol{x}, t)+p^{\prime}(\boldsymbol{x}, t)$, where coordinates and times are $(\boldsymbol{x}, t)$. With decomposition the Navier-Stokes equation is then transformed into Reynolds-averaged Navier - Stokes equations, where the Reynolds stress ten- 
sor $\boldsymbol{\tau}=-\rho \overline{\boldsymbol{u}^{\prime} \otimes \boldsymbol{u}^{\prime}}=-\rho \lim _{T \rightarrow \infty} \frac{1}{T} \int_{t}^{t+T}\left(\boldsymbol{u}^{\prime} \otimes \boldsymbol{u}^{\prime}\right) d t$ is introduced, where $T$ is the period of time over which the averaging takes place and must be sufficiently large to give meaningful averages. Reynolds stress is apparent stress owing to the fluctuating velocity field $\boldsymbol{u}^{\prime}$.

As we know, the Navier-Stokes momentum equation is $\rho \boldsymbol{u}_{, t}+\boldsymbol{\nabla} \cdot \boldsymbol{\Pi}=0$, continuity equation of incompressible flow is $\boldsymbol{\nabla} \cdot \boldsymbol{u}=0$, where the energy-moementum tensor given by $\boldsymbol{\Pi}=p \boldsymbol{I}+\rho \boldsymbol{u} \otimes \boldsymbol{u}-\mu(\boldsymbol{\nabla} \boldsymbol{u}+\boldsymbol{u} \boldsymbol{\nabla})$, dynamic viscosity $\mu$, gradient operator $\boldsymbol{\nabla}=\boldsymbol{e}_{i} \partial_{i}$, base vector in the i-coordinate $\boldsymbol{e}_{i}$, and tensor product $\otimes$. By introducing the Reynolds decomposition and averaging operation, we have the Reynolds equations and continuity equation of the mean velocity as follows, respectively: $\rho \overline{\boldsymbol{u}}_{, t}+\rho \boldsymbol{\nabla} \cdot(\overline{\boldsymbol{u}} \otimes \overline{\boldsymbol{u}})+\boldsymbol{\nabla} \bar{p}=\mu \boldsymbol{\nabla}^{2} \overline{\boldsymbol{u}}-\rho \boldsymbol{\nabla} \cdot\left(\overline{\boldsymbol{u}^{\prime} \otimes \boldsymbol{u}^{\prime}}\right)$ and $\boldsymbol{\nabla} \cdot \overline{\boldsymbol{u}}=0$.

For a general three-dimensional flow, there are four independent equations governing the mean velocity field; namely three components of the Reynolds equations together with one mean continuity equation. However, these four equations contain more than four unknowns. In addition to $\overline{\boldsymbol{u}}$ and $\bar{p}$ (four quantities), there are also the Reynolds stresses, people believe the Reynolds stresses has six unknowns (in the later, we will show that the Reynolds stresses have only three unknowns). Now we have ten unknowns and four equations. Thats is why people believe the Reynolds equations are unclosed. This is a manifestation of the closure problem.

In 1940, P.-Y. Chou [30, 31] pointed out that because the Navier-Stokes equations are the basic dynamical equations of fluid motion, it is insufficient to consider only the mean turbulent motion. The turbulent fluctuations are as important as the mean motion and the equations for turbulent fluctuations also need to be considered.

Subtracting the mean motions from the Navier-Stokes equation and continuity equation, Chou [30, 31] obtained the equations of the turbulence fluctuations $\rho \overline{\boldsymbol{u}}_{, t}^{\prime}+\rho \boldsymbol{\nabla}$. $\left(\overline{\boldsymbol{u}} \otimes \boldsymbol{u}^{\prime}+\boldsymbol{u}^{\prime} \otimes \overline{\boldsymbol{u}}+\boldsymbol{u}^{\prime} \otimes \boldsymbol{u}^{\prime}\right)+\nabla p^{\prime}=\mu \nabla^{2} \boldsymbol{u}^{\prime}+\rho \boldsymbol{\nabla} \cdot\left(\overline{\boldsymbol{u}^{\prime} \otimes \boldsymbol{u}^{\prime}}\right)$ and $\boldsymbol{\nabla} \cdot \boldsymbol{u}^{\prime}=0$. After having the above fluctuation equations, Chou [30, 31] introduced hierarchy of equations for velocity fluctuation correlations, however, any velocity correlation equation of a given order always obtains an unknown velocity correlation of one higher order, i.e. all hierarchy are still not closed.

Although Chou [31] mentioned that the rigorous way of treating the turbulence problem is probably to solve the Reynolds' equations of mean motion and the equations of turbulent fluctuation simultaneously. However, from the presentation of [31] and all his subsequent publications
[32-36], we noticed that Chou together with all other researchers $[1-8,10-16]$ did not realise that the fluctuation equations together with the mean equations already can form a closed equations system. The turbulence closure problem has not been solved even since. To close the so-called closure problem, a number of turbulence models on the Reynolds stress modelling have been proposed $[1-8,10-16]$, however no one is universal.

Regarding the closure issue, the current common view is that there are six unknowns in the Reynolds stress tensor, namely $\tau_{11}, \tau_{12}, \tau_{13}, \tau_{22}, \tau_{23}, \tau_{33}$; while we have a completely different perspective that we believe the Reynolds stress tensor has only three unknowns, namely the velocity fluctuation components $u_{i}^{\prime}(i=1,2,3)$. So that the mean and fluctuation turbulence equations are closed, and no additional restrictions are needed. The so-called turbulence closure problem is not an issue at all $[37,38]$.

To support this claim, let's us introduce two Lemma as follows

Lemma 1 Giving two real functions, $a(\boldsymbol{x}, t), b(\boldsymbol{x}, t)$, we can construct a 2nd order symmetric matrix function $M(\boldsymbol{x}, t)$ by their multiplication

$$
M(\boldsymbol{x}, t)=\left[\begin{array}{ll}
a a & a b \\
b a & b b
\end{array}\right]
$$

since $a b=b a$. Although $M$ has three independen$t$ elements, namely $a a, a b, b b$, however it is clear that there are only two independent parameters, namely, $a(\boldsymbol{x}, t), b(\boldsymbol{x}, t)$, in the matrix $M(\boldsymbol{x}, t)$. In other words, if we know $a(\boldsymbol{x}, t), b(\boldsymbol{x}, t)$, the matrix $M(\boldsymbol{x}, t)$ can be defined completely.

Similarly, giving three real number, $a, b, c$, we can construct a 3nd order symmetric matrix $N(\boldsymbol{x}, t)$ by their multiplication

$$
N(\boldsymbol{x}, t)=\left[\begin{array}{lll}
a a & a b & a c \\
b a & b b & b c \\
c a & c b & c c
\end{array}\right]
$$

since $a b=b a, a c=c a, b c=c b$. Although $M$ has six independent elements, namely $a a, a b, a c, b b, b c, c c$, however it is clear there are only three independent parameters, namely, $a, b, c$, in the matrix $N(\boldsymbol{x}, t)$.

Lemma 2 Giving two real functions, $a(\boldsymbol{x}, t), b(\boldsymbol{x}, t)$, we can defined a 2nd order symmetric matrix function $A(\boldsymbol{x}, t)$ as follows

$$
A(\boldsymbol{x}, t)=\lim _{T \rightarrow \infty} \frac{1}{T} \int_{t}^{t+T}\left[\begin{array}{ll}
a a & a b \\
b a & b b
\end{array}\right] d t
$$


where $a b=b a$. Although $A(\boldsymbol{x}, t)$ has three independent elements, namely $a a, a b, b b$, however it is clear that there are only two independent parameters, name$l y, a(\boldsymbol{x}, t) b(\boldsymbol{x}, t)$, in the matrix $A(\boldsymbol{x}, t)$. In other words, if we know a $(\boldsymbol{x}, t), b(\boldsymbol{x}, t)$, the matrix $A(\boldsymbol{x}, t)$ can be defined completely.

Similarly, giving three real functions, $a(\boldsymbol{x}, t), b(\boldsymbol{x}, t), c(\boldsymbol{x}, t), \quad$ we can construct a 3nd order symmetric matrix $B(\boldsymbol{x}, t)$ by their multiplication

$$
B(\boldsymbol{x}, t)=\lim _{T \rightarrow \infty} \frac{1}{T} \int_{t}^{t+T}\left[\begin{array}{ccc}
a a & a b & a c \\
b a & b b & b c \\
c a & c b & c c
\end{array}\right] d t,
$$

where $a b=b a, a c=c a, b c=c b$. Although $B(\boldsymbol{x} . t)$ has six independent elements, namely $a a, a b, a c, b b, b c, c c$, however it is clear there are only three independent parameters, namely, $a(\boldsymbol{x}, t), b(\boldsymbol{x}, t), c(\boldsymbol{x}, t)$, in the matrix $B(\boldsymbol{x}, t)$.

This is the exact situation in the turbulence modelling of the Reynolds stress tensor, if we consider the $a(\boldsymbol{x}, t), b(\boldsymbol{x}, t), c(\boldsymbol{x}, t)$ as the components of velocity fluctuations $u_{i}(i=1,2,3)$, respectively. From the Lemma 2 , we can see the Reynolds stress tensor has only three instead of six unknowns. This can be proved easily as follows: the 3D Reynolds stress tensor can be defined by

$$
\begin{aligned}
\boldsymbol{\tau} & =-\rho \overline{\boldsymbol{u}^{\prime} \otimes \boldsymbol{u}^{\prime}}=-\rho \overline{u_{i}^{\prime} \boldsymbol{e}_{i} \otimes u_{j}^{\prime} \boldsymbol{e}_{j}}=-\rho \overline{u_{i}^{\prime} u_{j}^{\prime}} \boldsymbol{e}_{i} \otimes \boldsymbol{e}_{j} \\
& =-\rho \lim _{T \rightarrow \infty} \frac{1}{T} \int_{t}^{t+T}\left[u_{i}^{\prime} u_{j}^{\prime} \boldsymbol{e}_{i} \otimes \boldsymbol{e}_{j}\right] d t \\
& =-\rho \lim _{T \rightarrow \infty} \frac{1}{T} \int_{t}^{t+T}\left[u_{1}^{\prime} u_{1}^{\prime} \boldsymbol{e}_{1} \otimes \boldsymbol{e}_{1}+u_{1}^{\prime} u_{2}^{\prime} \boldsymbol{e}_{1} \otimes \boldsymbol{e}_{2}\right. \\
& +u_{1}^{\prime} u_{3}^{\prime} \boldsymbol{e}_{1} \otimes \boldsymbol{e}_{3}+u_{2}^{\prime} u_{1}^{\prime} \boldsymbol{e}_{2} \otimes \boldsymbol{e}_{1}+u_{2}^{\prime} u_{2}^{\prime} \boldsymbol{e}_{2} \otimes \boldsymbol{e}_{2} \\
& +u_{2}^{\prime} u_{3}^{\prime} \boldsymbol{e}_{2} \otimes \boldsymbol{e}_{3}+u_{3}^{\prime} u_{1}^{\prime} \boldsymbol{e}_{3} \otimes \boldsymbol{e}_{1}+u_{3}^{\prime} u_{2}^{\prime} \boldsymbol{e}_{3} \otimes \boldsymbol{e}_{2} \\
& \left.+u_{3}^{\prime} u_{3}^{\prime} \boldsymbol{e}_{3} \otimes \boldsymbol{e}_{3}\right] d t
\end{aligned}
$$

and fluctuation velocity convective terms

$$
\begin{aligned}
& \overline{\boldsymbol{u}^{\prime} \cdot \boldsymbol{\nabla} \boldsymbol{u}^{\prime}}=\overline{u_{i}^{\prime} \boldsymbol{e}_{i} \cdot\left[\boldsymbol{e}_{k} \partial_{k} \otimes\left(u_{j}^{\prime} \boldsymbol{e}_{j}\right)\right]} \\
& =\overline{u_{i}^{\prime} u_{j, k}^{\prime}} \boldsymbol{e}_{i} \cdot\left(\boldsymbol{e}_{k} \otimes \boldsymbol{e}_{j}\right)=\overline{u_{i}^{\prime} u_{j, k}^{\prime}}\left(\boldsymbol{e}_{i} \cdot \boldsymbol{e}_{k}\right) \boldsymbol{e}_{j} \\
& =\overline{u_{i}^{\prime} u_{j, k}^{\prime}} \delta_{i k} \boldsymbol{e}_{j}=\overline{u_{i}^{\prime} u_{j, i}^{\prime}} \boldsymbol{e}_{j} \\
& =\left(\lim _{T \rightarrow \infty} \frac{1}{T} \int_{t}^{t+T} u_{i}^{\prime} u_{j, i}^{\prime} d t\right) \boldsymbol{e}_{j} .
\end{aligned}
$$

The formulations in Eq. $(1,2)$ reveal that the Reynolds stress tensor $\boldsymbol{\tau}=-\rho \overline{\boldsymbol{u}^{\prime} \otimes \boldsymbol{u}^{\prime}}$ and fluctuation velocity convective terms can be fully calculated by three independent components of fluctuation velocity $u_{1}^{\prime}, u_{2}^{\prime}, u_{3}^{\prime}$. In other words, the Reynolds stress tensor has only three unknowns rather than six unknowns.
All mistakes regarding the numbers of unknown quantities stem from misunderstandings of the Reynolds stress tensor, $\boldsymbol{\tau}=-\rho \overline{\boldsymbol{u}^{\prime} \otimes \boldsymbol{u}^{\prime}}=-\rho \lim _{T \rightarrow \infty} \frac{1}{T} \int_{t}^{t+T}\left(\boldsymbol{u}^{\prime} \otimes \boldsymbol{u}^{\prime}\right) d t$, all literature state the symmetric Reynolds stress tensor has six unknowns. However, the Reynolds stress tensor is actually not an arbitrary 2nd order tensor with six independent elements, but each of the element is made of the bi-product of fluctuation velocity component. For three dimensional flow, we can only have three components of fluctuation velocity $\boldsymbol{u}^{\prime}$ as unknowns. It means that the Reynolds stress tensor has only three unknowns, namely $u_{1}^{\prime}, u_{2}^{\prime}, u_{3}^{\prime}$. For two dimensional flow, of course, the $2 \mathrm{D}$ Reynolds stress tensor has only two unknowns, namely $u_{1}^{\prime}, u_{2}^{\prime}$.

With the above understanding, we can write down the closed turbulence equations as follows

$$
\begin{aligned}
& \rho \overline{\boldsymbol{u}}_{, t}+\rho \boldsymbol{\nabla} \cdot(\overline{\boldsymbol{u}} \otimes \overline{\boldsymbol{u}})+\boldsymbol{\nabla} \bar{p}=\mu \boldsymbol{\nabla}^{2} \overline{\boldsymbol{u}} \\
& -\rho \lim _{T \rightarrow \infty} \frac{1}{T} \int_{t}^{t+T} \boldsymbol{\nabla} \cdot\left(\boldsymbol{u}^{\prime} \otimes \boldsymbol{u}^{\prime}\right) d t, \\
& \rho \overline{\boldsymbol{u}}^{\prime}, t+\rho \boldsymbol{\nabla} \cdot\left(\overline{\boldsymbol{u}} \otimes \boldsymbol{u}^{\prime}+\boldsymbol{u}^{\prime} \otimes \overline{\boldsymbol{u}}+\boldsymbol{u}^{\prime} \otimes \boldsymbol{u}^{\prime}\right)+\boldsymbol{\nabla} p^{\prime} \\
& =\mu \boldsymbol{\nabla}^{2} \boldsymbol{u}^{\prime}+\rho \lim _{T \rightarrow \infty} \frac{1}{T} \int_{t}^{t+T} \boldsymbol{\nabla} \cdot\left(\boldsymbol{u}^{\prime} \otimes \boldsymbol{u}^{\prime}\right) d t, \\
& \nabla \cdot \overline{\boldsymbol{u}}=0, \\
& \boldsymbol{\nabla} \cdot \boldsymbol{u}^{\prime}=0 .
\end{aligned}
$$

Applying the divergence operation $\nabla$ on both sides of the Eqs.(3,4), we can obtain equations for mean pressure: $\boldsymbol{\nabla}^{2} \bar{p}=-\rho \boldsymbol{\nabla} \cdot(\overline{\boldsymbol{u}} \cdot \boldsymbol{\nabla} \overline{\boldsymbol{u}})-\rho \lim _{T \rightarrow \infty} \frac{1}{T} \int_{t}^{t+T} \boldsymbol{\nabla} \cdot\left(\boldsymbol{u}^{\prime} \cdot \boldsymbol{\nabla} \boldsymbol{u}^{\prime}\right) d t$, equation for fluctuation velocity $\nabla^{2} p^{\prime}=-\rho \boldsymbol{\nabla} \cdot\left[\overline{\boldsymbol{u}} \cdot \boldsymbol{\nabla} \boldsymbol{u}^{\prime}+\right.$ $\left.\boldsymbol{u}^{\prime} \cdot \boldsymbol{\nabla} \overline{\boldsymbol{u}}+\boldsymbol{u}^{\prime} \cdot \boldsymbol{\nabla} \boldsymbol{u}^{\prime}\right]+\rho \lim _{T \rightarrow \infty} \frac{1}{T} \int_{t}^{t+T} \boldsymbol{\nabla} \cdot\left(\boldsymbol{u}^{\prime} \cdot \nabla \boldsymbol{u}^{\prime}\right) d t$

Amazingly the integral-differential equations in Eqs. $(3,4,5,6)$ have exactly eight equations, which equal to the numbers of unknowns, namely, three components of mean velocity $\overline{\boldsymbol{u}}$, three components of fluctuation velocity $\boldsymbol{u}^{\prime}$, one mean pressure $\bar{p}$ and one fluctuation pressure $p^{\prime}$. That is why the integral-differential equations system of Eqs. $(3,4,5,6)$ are closed!

Therefore, our statement can be expressed as follows: The Reynolds Navier-Stokes turbulence equations of incompressible flow in Eqs. $(3,4,5,6)$ are closed rather than unclosed. This understanding can definitely provide a better guideline in the modelling of turbulence [37-39].

It is my great pleasure to have shared and discussed some of the above with Michael Sun from Bishops Diocesan College, whose pure and direct scientific sense inspired me. 
[1] Bradshaw P. An Introduction to Turbulence and Its Measurement. Pergamon Press, New York (1971).

[2] Tennekes H. and Lumley, J.L. A First Course in Turbulence. Cambridge: The MIT Press (1972).

[3] Lesilie D.C. Developments in the Theory of Turbulence. Clarendon Press, Oxford (1973).

[4] Townsend A.A. The Structure of Turbulent Shear Flow 2nd ed., Cambridge University Press, New York (1976).

[5] Lesieur M. Turbulence in Fluids. 2nd ed. Kluwer, Dordrecht (1990).

[6] Wilcox D.C. Turbulence Modeling for CFD. D C W Industries (1993).

[7] Pope S.B. Turbulent Flows. Cambridge University Press, Cambridge (2000).

[8] Davidson P.A. Turbulence. Oxford University Press, Oxford (2004).

[9] Hof B. Experimental Observation of Nonlinear Traveling Waves in Turbulent Pipe Flow. Science 305, 1594 (2004).

[10] Falkovich G. and Sreenivasan K.R. Lessons from hydrodynamic turbulence. Physics Today, 43-49 (April 2006).

[11] Frisch U. Turbulence: The Legacy of A.N. Kolmogorov. Cambridge University Press, Cambridge (2008).

[12] Marusic I. Mathis R. and Hutchins N. Predictive model for wall-bounded turbulent Flow. Science 329, 193 (2010).

[13] Smits A.J., McKeon B.J. and Marusic I. High - Reynolds Number Wall Turbulence, Annu. Rev. Fluid Mech. 43, 353 (2011).

[14] Davison P.A. et al. A Voyage Through Turbulence. Cambridge: Cambridge University Press (2011).

[15] Suri B., Tithof J. R., Grigoriev R.O. and Schatz M.F. Forecasting Fluid Flows Using the Geometry of Turbulence. Phys. Rev. Lett. 118, 114501 (2017).

[16] Cardesa et al. The turbulent cascade in five dimensions. Science 35, $782-784$ (2017).

[17] http://www.claymath.org/millennium-problems/navierstokes-equation.

[18] Castelvecchi D. On the trial of turbulence. Nature, 548:382 (2017).

[19] Kolmogorov A.N. The local structure of turbulence in incompressible viscous fluid for very large Reynolds number. Dokl. Akad. Nauk SSSR, 30:299-303 (1941a) (reprinted in Proc.R.Soc.Lond. A, 434,9-13, 1991).

[20] Kolmogorov A.N. On degeneration (decay) of isotropic turbulence in an incompressible visous liquid. Dokl. Akad. Nauk SSSR, 31:538-540 (1941b).

[21] Kolmogorov A.N. Dissipation of energy in locally isotropic turbulence. Dokl.Akad. Nauk SSSR, 32:1618 (1941c).(reprinted in Proc.R.Soc.Lond. A, 434,15-17, 1991).

[22] Sreenivasan K.R. On the scaling of the turbulence energy dissipation rate. Physics of Fluids, 27,5:1048-1051
(1984).

[23] Sun B.H.. The temporal scaling laws of compressible turbulence. Modern Physics Letters B. 30,(23) 1650297 (2016).

[24] Sun B.H. Scaling laws of compressible turbulence. Appl. Math. Mech.-Engl. Ed. 38: 765(2017).

[25] She Z.S. and Lévêque E. Universal scaling laws in fully developed turbulence. Phys. Rev. Lett. 72,336(1994) .

[26] Lorenz E.N. Deterministic non-periodic flow. J. Atmos Sci. 20:130 - 41(1963).

[27] Benzi R, Paladin P., Parisis G. and Vulpiani A. On the multifractal nature of fully developed turbulence and chaotic systems. J. Phys. A: Math. Gen. 17:35213531(1984).

[28] Orszag S.A. and Patterson G. S. Jr. Numerical simulation of three-dimensional homogeneous isotropic turbulence. Phys. Rev. Lett. 28,76(1972).

[29] Reynolds O. On the dynamical theory of incompressible viscous fluids and the determination of the criterion. Philos. Trans. R. Soc. 186:123 - 164(1895).

[30] Chou P-Y. On an extension of Reynolds' method of finding apparent stress and the nature of turbulence. Chin. J. Phys. 1-53 (1940).

[31] Chou P-Y. On velocity correlations and the solutions of the equations of turbulent fluctuation. Q. Appl. Math. 111(1):38-54(1945).

[32] Chou P-Y, Cai S-T. The vorticity structure of homogeneous isotropic turbulence in its final period of decay. Acta Mech. Sinica 1(1): 3-14(1957).

[33] Chou P-Y, Huang Y-N. On the statistical vorticity structure theory of homogeneous isotropic turbulence. Sci. Sinica XVIII(2): 199-222(1975).

[34] Chou P-Y. On the condition of pseudo-similarity and the theory of turbulence. Sci. Sinica XXVIII(4): 405(1985)

[35] Chou P-Y, Chen S-Y. On the theory of turbulence for incompressible fluid. Sci. Sinica (4): 369-80 (1987).

[36] Chou P-Y. and Chou, R.L. 50 years of turbulence research in China. Annu. Rev. Fluid Mech. 27:1-15(1995).

[37] Sun B. The Reynolds Navier-Stokes turbulence equations of incompressible flow are closed rather than unclosed. Preprints 2018, 2018060461 (doi: 10.20944/preprints201806.0461.v1).

[38] Sun B. The Reynolds Navier-Stokes turbulence equations of incompressible flow are closed rather than unclosed. Preprints 2018, 2018060461 (doi: 10.20944/preprints201806.0461.v2).

[39] Sun B. A Novel Simplification of the ReynoldsChou-Navier-Stokes Turbulence Equations of Incompressible Flow. Preprints 2018, 2018070030 (doi: 10.20944/preprints201807.0030.v1). 